\title{
COVID outbreak is changing our practices of perinatal psychiatry
}

\section{Florence Gressier $^{1,2} \cdot$ Antonia Mezzacappa $^{1,2} \cdot$ Pierre-Alexandre Lasica $^{1,2} \cdot$ Corine Fourcade $^{1} \cdot$ Emmanuelle Corruble $^{1,2}$}

Received: 15 April 2020 / Accepted: 15 May 2020 / Published online: 28 May 2020

(C) Springer-Verlag GmbH Austria, part of Springer Nature 2020

\section{Dear Editor,}

COVID outbreak is leading us to reconsider perinatal psychiatry management.

Perinatal depression affects more than $15 \%$ of women (Shorey et al. 2018), and suicide is the leading cause of perinatal death in high-socioeconomic countries (Oates 2003).

With the COVID epidemic, obstetric consultations are limited, sometimes with only teleconsultation (Dashraath et al. 2020). Future fathers do not attend the prenatal ultrasound and will only meet their baby at birth, even a few days later. Hospital visits are prohibited, even in the maternity ward. Fathers are just allowed in the delivery room, but not in the case of C-section or COVID-19 symptoms.

The COVID epidemic is changing practices surrounding birth, as well as those surrounding death and mourning.

How does motherhood look in these conditions? How to help women at a time when the construction of family bonds is crucial?

To avoid contamination, psychiatric consulting in the maternity ward is most often limited to emergencies. When done, it is through a mask, for the professional and sometimes also for the patient, making facial expressions difficult to interpret. Child Protection Services also limit their actions, with the consequences of difficult situations being more complex and sometimes dangerous for the mother and/or the child.

This article is part of the Topical Collection on Women's mental health during the Covid-19 pandemic

Edited by: Anita Riecher-Rössler

Florence Gressier

florence.gressier@aphp.fr

1 Department of Psychiatry, Bicêtre University Hospital, Assistance Publique-Hôpitaux de Paris, Hôpitaux Universitaires Paris Sud, 78 rue du Général Leclerc, 94275 Le Kremlin Bicêtre, France

2 UVSQ, CESP, Inserm UMR1178, Faculté de Médecine Paris-Sud, Université Paris-Saclay, Univ Paris-Sud, 94275 Le Kremlin Bicêtre, France
The epidemic is changing the already particular practices of perinatal psychiatry: a new form of multidisciplinary support has to be imagined and created.

Considering the state of knowledge and the potential risk for the baby, most psychiatric mother-baby units in France had to temporarily suspend their admissions. Information is needed to raise awareness about perinatal psychiatric disorders among women and their families more than ever before.

The question on mood, anxiety, suicidal ideation, and substance abuse must also be systematically asked by any health professional as multidisciplinary face-to-face consultations are not recommended. The Edinburgh Postpartum Depression Scale (EPDS) can help professionals not familiar with psychiatry to screen at-risk patients (Cox 2019), and perinatal psychiatric teleconsultation can increase access to perinatal psychiatry expertise (Wichman et al. 2019). The identification of at-risk situations must lead to specialized consultation. Teleconsultation should be preferred over phone to identify facial expressions. Information should be shared among different healthcare professionals in a multidisciplinary manner, by email or, if possible, in the most sensitive situations, by videoconference.

Throughout this period of confinement, follow-up should be as close as possible. We offer home psychiatric video consultations as often as needed, at least once a week for patients at risk or who have just come out of maternal-infant hospitalization. Prescriptions can be sent through the telepsychiatry site. Continuity is also maintained with the unit pediatrician, nurses, and childcare workers. Women also have a phone number and an email to ask practical questions about baby care and development (feeding: breastmilk, formula, and food diversification; bath; sleep; and vaccinations) whenever they want. These emails are read several times a day. A reply is given the same day or the next day by email, phone, or teleconsultation, even on weekends. In case of emergencies, families can refer to the on-call psychiatrist, present $24 \mathrm{~h}$ a day. Teleconsultation can also be useful to see the baby's environment and bonding. With the reduced professional help, assistance from the family is more important than ever: some home video consultations are conducted with the father, 
the family, and the baby/young child to ensure the quality of the links and interactions. A multidisciplinary video consultation could also be proposed with a psychiatrist, a pediatrician, and nurses if necessary. These young mothers also benefit from a care support by community pediatricians or the Protection Médicale Infantile-Maternal and Child Welfare (PMI) and childcare workers.

During this period of COVID-19 outbreak, it is essential that perinatal psychiatry workers continue to support pregnant women, young mothers, and their families using teleconsultation, phone, or web-based calls or e-mails. All professionals involved in the perinatal psychiatry network should work to improve the use of teleconsultation.

In the long term, the generalization of teleconsultation in perinatal psychiatry could allow observations of family interactions at home.

Authors' contributions Florence Gressier wrote the first draft. All the individuals included as authors of this letter contributed to and have approved the final manuscript.

\section{Compliance with ethical standards}

Conflict of interest The authors declare that they have no conflict of interest.

Ethics approval Not applicable.
Consent to participate Not applicable.

Consent for publication Not applicable.

\section{References}

Cox J (2019) Thirty years with the Edinburgh Postnatal Depression Scale: voices from the past and recommendations for the future. $\mathrm{Br} \mathrm{J}$ Psychiatry 214:127-129. https://doi.org/10.1192/bjp.2018.245

Dashraath P, Jing Lin Jeslyn W, Mei Xian Karen L, Li Min L, Sarah L, Biswas A, Arjandas Choolani M, Mattar C, Lin SL (2020) Coronavirus disease 2019 (COVID-19) pandemic and pregnancy. Am J Obstet Gynecol S0002-9378(20):30343-30344. https://doi. org/10.1016/j.ajog.2020.03.021

Oates M (2003) Suicide: the leading cause of maternal death. Br J Psychiatry 183:279-281. https://doi.org/10.1192/bjp.183.4.279

Shorey S, Chee CYI, Ng ED, Chan YH, Tam WWS, Chong YS (2018) Prevalence and incidence of postpartum depression among healthy mothers: a systematic review and meta-analysis. Psychiatry Res 104:235-248. https://doi.org/10.1016/j.jpsychires.2018.08.001

Wichman CL, Laszewski A, Doering JJ, Borchardt S (2019) Feasibility of model adaptations and implementation of a perinatal psychiatric teleconsultation program. Gen Hosp Psychiatry 59:51-57. https:// doi.org/10.1016/j.genhosppsych.2019.05.007

Publisher's note Springer Nature remains neutral with regard to jurisdictional claims in published maps and institutional affiliations. 